\title{
Modelling parking based trips
}

\author{
A. Levashev, A. Mikhailov \& I. Golovnykh \\ National Research Irkutsk State Technical University, Russia
}

\begin{abstract}
Nowadays, Russia is going through an era of rapid growth in private car ownership. This has resulted in new trends in Russian transportation planning practice. Previously in the USSR, private car ownership accounted for ten percent of the total passenger flow divided by the car occupancy. However, the rapid growth of private car ownership is only one aspect among many other significant changes in Russian socio-economic life. Consequently, Russian transportation planning has been challenged immensely. It is becoming necessary to forecast private car usage taking into account reasons for different journeys. Unfortunately, there are no current statistics in Russia that describe what we have defined as trip generation rates of different object types. Statistics collected during the Soviet era is included in urban planning manuals, but now this data is completely inconsistent with the current situation. The Transportation Laboratory of Irkutsk State Technical University launched regular investigations into the main indices of trip generation. The estimation of parking demand generation and parking accumulation is very laborious. In order to make parking surveys more effective the Laboratory proposed to collect video records from the security cameras of the parking facilities. The objective of the survey is to calculate the numbers of arriving and departing vehicles for each defined time period. The mathematical formula used to calculate parking duration is equal to the OD matrix estimation from traffic counts. The application of this method allows for the collation of all basic data, which is essential to calculate and forecast all factors of parking statistics. That makes possible to develop aggregated and disaggregated transport models including trip generation rates caused by different land use patterns, to plan shared parking and establish tariff policy applied to different parking lots.
\end{abstract}

Keywords: parking facility, parking demand estimation, parking duration. 


\section{Introduction}

The period between 1990 and the year 2000 might be recognized as the crisis period for transportation planning in the Russian Federation. Urban municipalities faced new economic and social realities as well as being immersed in current concerns they could not afford to develop long-term urban planning programs.

During the last decade the situation in Russia had been gradually changing. Municipalities displayed genuine interest in urbanism, with urban planning and transport infrastructure design definitely increasing. It can also be confirmed by the fact that Russia has recently become Europe's largest market for macro and micro simulation software.

The new economic and social trends are accompanied by social stratification and rapid growth of motorization. Levels of motorization in Russian cities vary widely from 0.22 to 0.50 cars per capita, but stable growth of private car ownership is the general trend in all Russian regions.

In this regard, Russian transportation and urban planners have a new challenge - the necessity to forecast the demand split between public and private transport.

\section{Statement of the problem}

In the former USSR analysis of public and private transport was calculated in a simple way. Regarding automobile rates of $0,08-0,100$ cars per capita it was generally supposed that almost 85-90 percent of overall journeys were served by a public transport, the other 10 percent of journeys were served by private transport. Car occupancy, if reasons for trips were not taken into consideration was considered always the same -1.5 persons per car. Central Urban Planning and Research Institute (TSNIIP) (leading urban research company in former Soviet Union) studied and systematized trip generation rates for different types of facilities. These data were published in 1978 in "Urban planner handbook" [1].

All the transport statistics from the previous economic and social period have become completely inappropriate because of new social and even travel behavior. These two aspects have been significantly changed lately. In spite of the fact that it is becoming more and more common in Russia to purchase transportation planning software, the level of transportation planners' skills still remains low. Probably, the absence of research on transportation planning during the ten year period (1990-2000) can be regarded as a principal reason for this. That period of time was sufficient to cause the decrease of professional skills in this area. The most complicated thing in this regard is the initial stage, to be more exact, the analysis and forecast of transportation demand. In comparison with the former Soviet period, it has become more difficult to interview the population and private car owners in particular. However, there is another issue raised; it turns out to be a formidable challenge to convince the municipal 
authorities that analysis of travel demand is a worthwhile starting point for the transportation planning process.

Such circumstances took our interest as disaggregated models of transportation demand. Analysis by Russian universities and research companies' showed that both statistical trip generation and other models are vacant subjects. Nowadays, former researcher and urban planning company (TSNIIP) is not engaged in this. Thus Transportation Laboratory of National Research Irkutsk State Technical University has chosen to conduct research of trip generation and disaggregated model as essential areas of study. This includes both $\mathrm{PhD}$ thesis and collecting background material for practical use. Gradually the entire object list mentioned in the former Soviet manual "Urban planner handbook” [1] and the latest version of “Trip Generation Handbook” has been coming under review.

Large shopping areas such as supermarkets and hypermarkets with free parking zones are the most complicated areas for transport demand estimation. In order to give a comprehensive description of such areas it's necessary to have a list of certain features: daily parking demand, average parking duration, parking accumulation, parking turnover etc.

Video processing from security cameras is one of the possible ways to collect comprehensive data. It gives an opportunity to obtain detailed information concerning the number of cars that come to a parking lot and leave at certain time intervals. It is sufficient to estimate daily parking demand and peak periods. However, there are some problems with the estimation of parking characteristics. Unfortunately, in most cases the quality of the video isn't sufficient to identify the number of vehicles. So it seems important to find a mathematical basis for the calculation of parking duration, parking accumulation etc.

Therefore, the goal of the research has been defined as to develop a mathematical procedure for the calculation of parking duration. Without fixing the private numbers it will serve to count the numbers of arriving and departing vehicles over a certain time period.

It was intended that this task can be solved mathematically as a calculation of the OD matrix, to be more exact, as bus passenger OD matrix estimation using counting systems. In fact, the main idea is to change the origins and destinations by time intervals. In this respect OD matrix is calculated with time intervals of arrivals as origins and time intervals of departures as destinations.

\section{Model notification and specification}

It is possible to organize the information about the volumes of arriving and departing vehicles as the triangular matrix with nonzero diagonal (table 1) (where $x_{i j}$ - number of vehicles accessing parking lot during interval $i$ and egressing it during time interval $j ; k$ - number of time intervals; $O_{i}$ - number of vehicles accessing parking lot during interval $i ; D_{j}$ - number of vehicles egressing parking lot during interval $j ; N$ - total number of parking vehicles). Accordingly, the values $x_{i j}$ in cells at diagonal and above it are the number of vehicles entering parking lot at time interval $i$ and departing parking lot at time 
interval $j$. It was assumed that volumes of vehicles entering parking lot and leaving it are related as a gravity patterns and the point of estimating parking duration here is to calibrate the model from the observed numbers.

The proposed calculation procedure of parking duration is based on the calculation of an OD-matrix model developed and intensively used by the Transportation Laboratory of ISTU [2, 3]. The popular Least Square Estimator $\sum e_{i}^{2}$ of conventional regression is replaced by a robust calculation technique based on the minimization of error, defined as:

$$
\sum_{i=1}^{n}\left|e_{i}\right|=\sum_{i=1}^{n}\left|y_{i}-\hat{y}_{i}\right|^{v}
$$

where

$e_{i}$ - regression errors

$y_{i}$ - measured traffic flow volumes $i, i=1,2, \ldots, n$;

$n$ - number of links with measured traffic volumes;

$v$ - parameter, which value is bounded as $1 \leq v<2$.

Table 1: Vehicles' circulation at parking lot description as matrix.

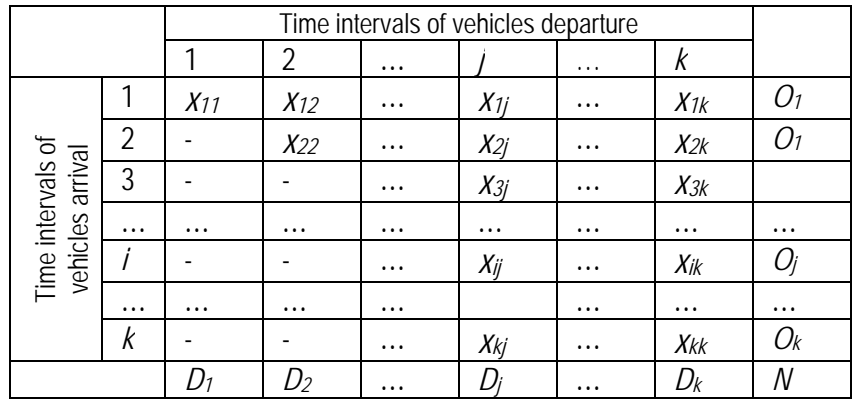

Calculation of trip matrices from traffic counts has a long history and many scientists mentioned this matter offering different estimation techniques [4-8]. The choice of robust regression, eqn. (1), for OD-matrix estimation is caused by the fact that we mostly process data from sources where this data has been positively skewed. Therefore, the assumption of normally distributed regression errors $e_{i}$ is not always feasible.

Particular case ( $v=1)$ of regression, eqn. (1), is the Least Absolute Error (or Least Absolute Deviations) which is used in our estimation routine

$$
\sum_{i=1}^{n}\left|e_{i}\right|=\sum_{i=1}^{n}\left|y_{i}-\sum_{j=1}^{m} a_{i j} x_{j}\right|
$$

where

$e_{i}-$ regression errors;

$y_{i}$ - measured traffic flow volumes at link $i, i=1,2, \ldots, n$;

$n$ - number of links with measured traffic volumes;

$a_{i j}=1$, if link $i$ and $j$ are correspondence $x_{j}$ incident and 0 otherwise (element of path-flow incidence matrix $\boldsymbol{A}$ ); $j=1,2, \ldots, m$;

$m$ - size of OD-matrix reordered as vector. 
The Least Absolute Errors estimation, eqn. (2), is considered as a procedure for linear optimization where the function object is:

$$
\sum_{i=1}^{n}\left(g_{i}+h_{i}\right)
$$

subject to the constraints:

$$
\begin{gathered}
g_{i}=\max \left|0, e_{i}\right| \geq 0, \\
h_{i}=\min \left|-e_{i}, 0\right| \geq 0, \\
\boldsymbol{A x}+\boldsymbol{g}-\boldsymbol{h}=\boldsymbol{y} .
\end{gathered}
$$

Here, $g_{i}$ and $h_{i}$ are $i$ related positive and negative deviation respectively $e_{i}$ respectively.

In order to estimate vector $\boldsymbol{x}$ of dimension $m \times 1$ it is significant to solve the linear programming problem for the new vector $\boldsymbol{x} \mathbf{2}$ of dimension $(m+2 n) \times 1$. The objective function (3) includes only the part of the vector $\mathbf{x} 2$ which starting with index $m+1$. Thus the incidence matrix $\boldsymbol{A}$ is converted into matrix $\boldsymbol{A} 2$ of dimension $n \times(m+2 n)$, i.e. previous matrix is supplemented by two diagonal matrices $D 1$ and $D 2$ :

$$
A 2=\left[\begin{array}{cccccccccccc}
a_{11} & a_{12} & \ldots & a_{1 m} & 1 & 0 & \ldots & 0 & -1 & 0 & \ldots & 0 \\
a_{21} & a_{22} & \ldots & a_{2 m} & 0 & 1 & \ldots & 0 & 0 & -1 & \ldots & 0 \\
\ldots & \ldots & \ldots & \ldots & \ldots & \ldots & \ldots & \ldots & \ldots & \ldots & \ldots & 0 \\
a_{n 1} & a_{n 2} & \ldots & a_{n m} & 0 & 0 & 0 & 1 & 0 & 0 & \ldots & -1
\end{array}\right]
$$

Liner Programming problem, eqn. (3), is solving iteratively. The error vector $\boldsymbol{e}$ each time is calculated taking into account the OD-vector $\boldsymbol{x}$ obtained in the previous iteration. The elements $x_{m+1}, x_{m+2}, \ldots, x_{(m+2 n)}$ of vector $\boldsymbol{x} \mathbf{2}$ is forming according to the sign of the error $e_{i}$. According to all rearrangements mentioned above, the linear programming problem eqn. (3) has received a new formulation:

$$
\min \sum_{j=m+1}^{m+2 n} x_{j},
$$

subject to the constraints:

$$
\begin{gathered}
A 2 x \mathbf{2}=\boldsymbol{y}, \\
x l b_{i} \leq x_{i} \leq x u b_{i}, \\
x_{i} \geq 0 .
\end{gathered}
$$

The Linprog function of the Matlab Optimization Tools library is the appropriate solver of the task, eqn. (5):

where

$$
\mathrm{x}=\operatorname{linprog}\left(\mathrm{f}, A, b, v l b, v u b, x^{0}\right)
$$

$f$ - linear objective function vector;

A - matrix for linear equality constraints;

$b$ - vector for linear equality constraints;

$v l b$ - lower bound vector; 
$v u b$ - upper bound vector;

$x^{0}-$ initial point for $x$.

Required for Linear programming the target matrix $\mathrm{x} 0$ is calculated using the modified gravity model:

where

$$
x_{i j(n)}=O_{i} \frac{C_{j(n)} d_{i j}(t) O_{i}}{\sum_{i=1}^{k} C_{j(n)} d_{i j}(t) D_{j}}
$$

$x_{i j}(n)$ - number of vehicles accessing parking lot during interval $i$ and egressing it during time interval $j$;

$O_{i}$ - number of vehicles accessing parking lot during interval $i$;

$D_{j}$ - number of vehicles egressing parking lot during interval $j$;

$C_{i(n)}=$ value of calibration factor at iteration $n$;

$k$ - number of time intervals;

$d_{i j}(t)$ - calibration factor, i.e. parking duration distribution function.

The gravity model, eqn. (7), requires certain function $d_{i j}(t)$ of the distribution of parking duration which cannot be predetermined in case we use only video records without considering any other sources of information. Thereby, three different functions $d_{i j}(t)$ were examined in the estimation procedure, eqn. (8):

$$
d_{i j}(t)=1 ; d_{i j}(t)=1 / t ; d_{i j}(t)=1 / t^{2} .
$$

\section{Model performance test}

For the model verification we surveyed a large parking lot at "Southern Market" in Irkutsk (fig. 1). This parking lot was chosen because of its 4-lane driveway connection with the main street. Due to this it is a very convenient site for parking studies. The procedure involves manual registration of number plates as

\begin{tabular}{|c|c|c|c|}
\hline iv $101-\frac{5}{5}=$ & Time & Licenc & umbers \\
\hline & & Arrival & Departure \\
\hline & $9: 00$ & E603 & E603 \\
\hline & & M716 & \\
\hline & & $X 203$ & \\
\hline & & $\mathrm{B} 602$ & \\
\hline & $9: 10$ & B047 & M716 \\
\hline 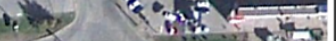 & & K993 & $\mathrm{X} 203$ \\
\hline & & A873 & \\
\hline$x^{2} \cdot e^{-2}$ & & $\mathrm{H} 690$ & \\
\hline
\end{tabular}
well as time of arriving and departing from a parking lot for each kind of vehicle

Figure 1: $\quad$ Scheme of parking lot at South Market in Irkutsk and license plate survey table. 
during the daytime (7.00 a.m.-8.00 p.m.). The research data was accepted as actual values.

Results of the research were arranged as two different combined sets of data. The first set was based on 30-minute intervals, whereas the second one on 60minute intervals. Taking into consideration combined data, it is essential to consider that actual parking duration deviates from the bounds of half-hour or hour intervals.

Correction of the parameters is calculated as the average difference between upper bounds of time intervals and actual parking durations as presented in fig. 1 ; for the case of 60 -minute intervals (figs 2 and 3 ).

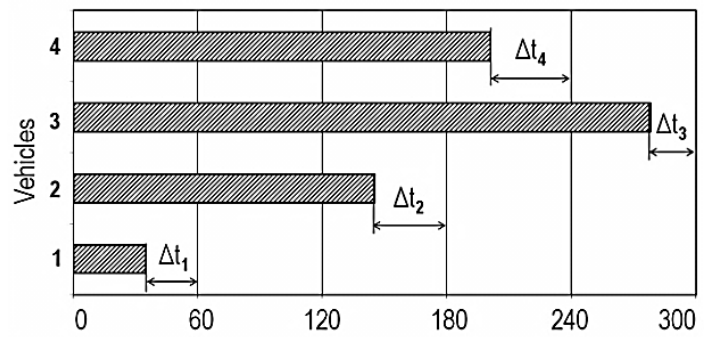

Figure 2: $\quad$ Estimation of average difference between upper bounds for time intervals and actual parking duration $\Delta \bar{t}=\sum \Delta t_{i} / N$ in the case of 60-minute intervals.

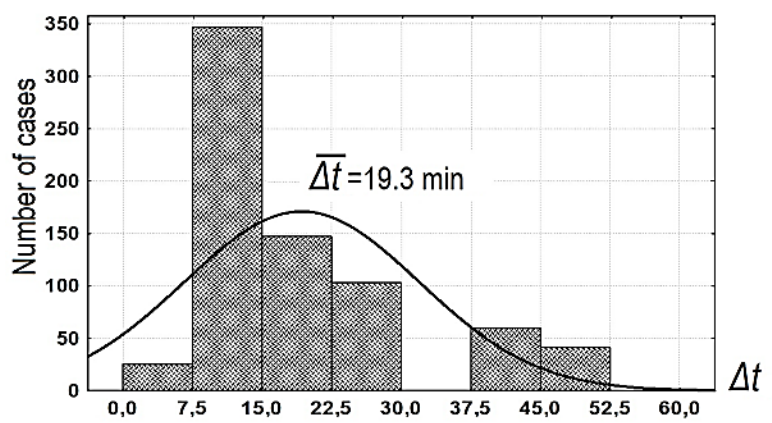

Figure 3: Actual distribution of difference between upper bounds for time intervals and actual parking duration in the case of 60-minute intervals.

Average parking duration defined for 60-minute intervals as:

$$
\bar{T}=\sum_{i}^{k} \sum_{j}^{k} T_{i j} / \sum_{i}^{k} \sum_{j}^{k} N_{i j}
$$

where

$i$ - index of interval when vehicle entering a parking lot, $i=1,2, \ldots, k$;

$k$ - number of time intervals; 
$j$ - index of interval when vehicle departing from a parking lot, $j \geq i$;

$\begin{array}{ll}\text { if } i \neq j & T_{i j}=N_{i j}[60(j-i)-\overline{\Delta t}] ; \\ \text { otherwise (matrix diagonal) } & T_{i j}=N_{i j}[60-\overline{\Delta t}] ;\end{array}$

$\overline{\Delta t}$ - average difference between upper bounds for time intervals and actual parking duration;

$N_{i, j}$ - number of vehicles entering a parking lot in a time interval $i$ and departing in a time interval $j$.

The first way to test the accuracy of the proposed model is to compare the actual and estimated numbers of vehicles entering a parking lot at the same interval $i$ and departing from a parking lot at different time intervals $j$, where $j=$ $i, i+1, \ldots, k$. Fig. 4 displays actual and estimated distribution patterns of departure for the time interval from 11 a.m. till 12 a.m. Student's tests of these samples proved that the estimated and surveyed data was not significantly different from each other.

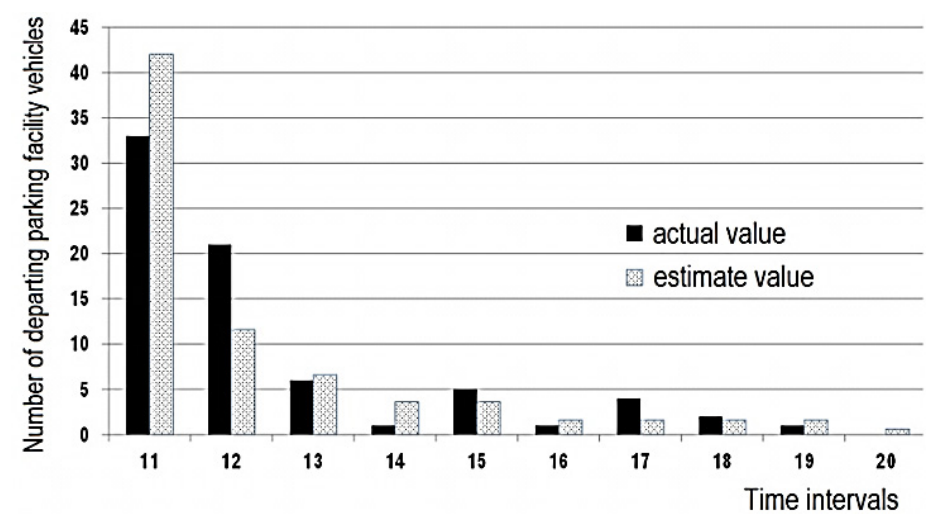

Figure 4: Comparison of actual and estimated values of departing vehicles which entered a parking lot in a time interval from 11 till 12 a.m.

Fig. 5 represents scatter plots of estimated and actual values of parking duration for both sets of 30 and 60 -minute intervals. Note that data combined for 60 -minute intervals give a much higher correlation $(\mathrm{R}=0.94)$.

The second way to check accuracy is to use certain measurements of distance between actual and estimated matrixes or the chi-square statistics. Then, the coefficient variation of the root-mean-square error was used to estimate the accuracy of the parking duration matrix:

$$
C V(R M S E)=\sqrt{\frac{\sum_{\mathrm{i}=1}^{\mathrm{n}}\left(x_{1, i}-x_{2, i}\right)^{2}}{n} / \bar{x}}
$$

where

$x_{1, i}$ and $x_{2, i}$ - values $i$ of cell in estimated and actual matrices respectively; $i=1,2, \ldots, n$; 
$n$ - total amount of cells in matrix;

$\bar{x}$ - average cell value in estimated matrix.
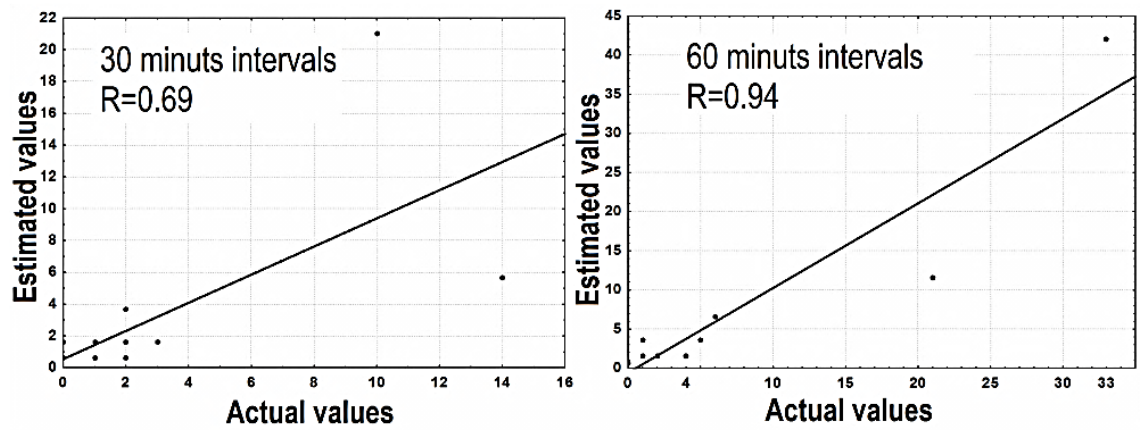

Figure 5: Comparison of actual and estimated parking duration in case of 30minute and 60-minute intervals.

The proposed model accuracy checking using CV(RMSE) statistics as well as scatter plots (fig. 5) shows that the combined interval of 60-minutes gives a more accurate estimation.

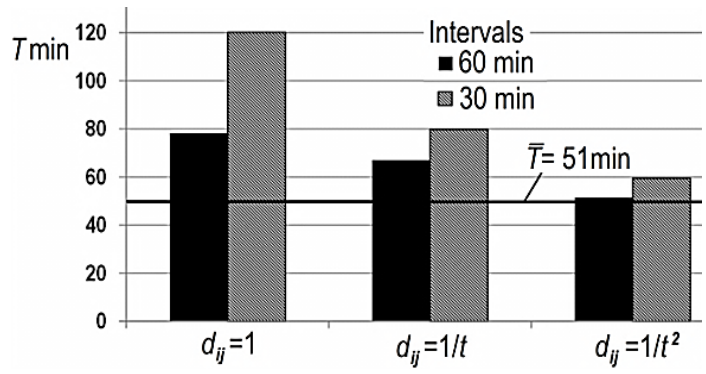

Figure 6: Accuracy of the average parking duration $\bar{T}$ estimation using different functions $d_{i j}(t)$.

All the routines that have been done showed that initial approximation $\mathrm{x} 0$, calculated by applying the function $d_{i j}(t)=1 / t^{2}$ gave the best results which were the closest to the survey data (fig. 6).

\section{Conclusion}

The proposed estimating technique has some margin for error confirmed by RMSE indicator. Nevertheless, it allows avoiding the procedure of manual vehicle counting and reduces the time required for surveying. As a result, time spent processing data in the given parking lot was reduced to $60 \%$.

The method is almost entirely based on video recording. In this regard we employed a low-cost method that allows for the analysis of all areas of a parking 
lot. Moreover, we have been actively using this method for the analysis of parking facilities.

Furthermore, we must establish detailed classification of parking facilities based on the parking accumulation. It will definitely help us to obtain a necessary set of procedures which are intended to improve the accuracy of the model. More than that, it is natural to assume that if the video records are replaced by data of traffic detectors, the process of parking survey will become fully automated.

\section{References}

[1] Urban planner handbook. Stroyizdat: Moscow, 369 p., 1978.

[2] Mikhailov, A.Y. and Golovnykh, I.M. Modern trends of urban highway networks design. Science: Novosibirsk, 267 p., 2004.

[3] Levashev, A.G. and Tebenkov, S.E. Estimation of traffic flows distribution at transport corridors. Irkutsk State Technical University Herald, 10, pp. 120-127, 2011.

[4] Cascetta, E. and Nguyen, S. A unified framework for estimating or updating origin/destination matrices from traffic counts. Transportation Research 22B, pp. 437-455. 1988.

[5] Chang, G. and Wu, J. Recursive Estimation of Time-Varying OriginDestination Flows from Traffic Counts in Freeway Corridors. Transportation Research-B, Vol. 28B, pp. 141-160, 1994.

[6] Bell, M.G.H. The Estimation of Origin-Destination Matrices by Constrained Generalised Least Squares, Transportation Research B, Vol.25B, No.1, pp. 13-22, 1991.

[7] Maher, M. Inferences on trip matrices from observations on link volumes: a Bayesian statistical approach. Transportation Research 20B, pp. 435-447, 1983.

[8] Van Der Zijpp, N.J. and Hamerslag, R. An Improved Kalman Filtering Approach to Estimate Origin-Destination Matrices for Freeway Corridors, Transportation Research Records, No.1443, pp. 54-64,1994. 